CORRESPONDENCE

\title{
Antibody responses to SARS-CoV2 vaccination in allogeneic hematopoietic stem cell transplant recipients
}

(C) The Author(s), under exclusive licence to Springer Nature Limited 2021

Bone Marrow Transplantation (2021) 56:3094-3096; https://doi.org/ 10.1038/s41409-021-01466-9

\section{TO THE EDITOR:}

Allogeneic hematopoietic stem cell transplantation (HSCT) is a potentially curative treatment for a wide range of hematological diseases but is still associated with significant infection-related morbidity and mortality related to post-transplant immunedeficiency. In the context of the COVID-19 pandemic, several studies have shown that allogeneic HSCT recipients have a higher risk of developing severe forms of COVID-19 and have a higher mortality rate compared to the general population [1, 2]. Induction of protective immunity through prophylactic vaccination is therefore critically important in this high-risk patient population. Although mRNA-based SARS-Cov-2 vaccines are highly effective in inducing protective responses in healthy individuals [3-5], whether they are able to induce protective immunity in allogeneic HSCT recipients is still unknown. In this study, we analyzed humoral responses to mRNA-based SARS-Cov-2 vaccines in 63 allogeneic HSCT recipients transplanted at Geneva University Hospitals (Supplementary Table 1). All patients signed an informed consent and the study was approved by the Geneva Ethical Committee (CCER-2021-01237). Patients were vaccinated between January and April 2021 with two doses of either the BNT162b2 (Pfizer) or the mRNA-1273 (Moderna) vaccines as part of our institutional priority vaccination program whose inclusion criteria included: patients who were at minimum 3 months and maximum 3 year since allogeneic HSCT; patients transplanted at more than 3 years post-transplant with GvHD requiring systemic immunosuppressive drugs; absence of Rituximab treatment in the previous 3 months; absence of ongoing steroid treatment at Prednisone $\geq 10 \mathrm{mg} /$ day. Blood samples were collected at a median of 67 (range 41-131) days from the first dose of vaccine and 38 (13-98) days after the administration of the second dose. In 32 patients, pre-vaccination blood samples collected at a median of $12(0-84)$ days before the administration of the first dose of vaccine were also available. Patients diagnosed with SARS-CoV-2 infection before vaccination or after vaccination but before serological analysis were excluded from the analysis. Vaccine-induced antibody responses against the SARS-CoV-2 spike protein (anti-S) were assessed in serum using the semi-quantitative Elecsys ${ }^{\varpi}$ Anti-SARSCoV-2 immunoassay (Roche). According to the manufacturer's instructions, the lower limit of detection of anti-S lgG was $0.4 \mathrm{U} / \mathrm{ml}$, the upper limit was $2500 \mathrm{U} / \mathrm{ml}$, and values above the cutoff of 0.8 $\mathrm{U} / \mathrm{ml}$ were considered positive. One value at the limit of positivity $(0.88 \mathrm{U} / \mathrm{ml})$ was considered negative after immunofluorescence did not confirmed the positivity. IgG against the SARS-CoV2 N- protein were measured in parallel to exclude immunization due to infection rather than vaccination.

We detected low levels anti-S IgG before vaccination in 4/32 available pre-vaccination samples, a result that was not confirmed by immunofluorescence validation nor by the detection of anti-N lgG, pointing to a false positive result rather than an exposure to the virus before vaccination. Forty-eight out of $63(76 \%)$ allogeneic HSCT recipients showed some degree of humoral response to vaccination based on anti-S IgG (Fig. 1a). Median levels of anti-S lgG were $815 \mathrm{U} / \mathrm{ml}$. Median age in our cohort was 54 (18-78) years. Given the previously reported impact of age in SARS-CoV-2 vaccine responses in the general population [3, 4], we assessed the impact of age on vaccine responses. We observed a trend not reaching statistical significance toward reduced anti-S IgG responses in patients older than 60 years $(16 / 25,64 \%$; median $183 \mathrm{U} / \mathrm{ml}$ ) compared to younger patients (32/38, 84\%; median $2347 \mathrm{U} / \mathrm{ml} ; p=0.055$; Fig. 1b). Fourty-six out of 63 (73\%) patients received mRNA-1273 and 17/63 (27\%) received BNT162b2 vaccines. No significant differences in the levels of anti-S IgG were detected between patients receiving BNT162b2 compared with patients receiving mRNA-1273 (data not shown). No grade 3-4 adverse events were reported after vaccination.

According to current EBMT and ASTCT recommendations, vaccination could be initiated as early as three months after HSCT if the transmission rate in the surrounding society is high (ASHASTCT General Principles of COVID-19 Vaccines for Immunocompromised Patients, Version 3.0; April 5, 2021; EBMT recommendations COVID-19 vaccines, Version 6.0, May 27, 2021). To improve the chances of response, the EBMT recommendations defined it as logical to wait until six months after transplantation if transmission in the surrounding society is well controlled. We assessed the potential impact of time since HSCT on antibodies responses in our patient's cohort. The first vaccine dose was administered at a median of $14(3-150)$ months after transplantation. We observed significantly lower anti-S lgG responses in patients receiving the first vaccine dose within 6 months since transplantation $(6 / 13$, $46 \%$; median $0.88 \mathrm{U} / \mathrm{ml}$ ) compared with patients vaccinated after 6 months post-HSCT $(42 / 50,84 \%$; median $2500 \mathrm{U} / \mathrm{ml} ; p=0.0016$; Fig. 1b). Moreover, EBMT recommendations define reasonable criteria to postpone COVID-19 vaccination the presence of uncontrolled acute GVHD grades III-IV, the administration of anti-CD20 antibodies during the past six months and recent therapy with anti-thymocyte globulin (ATG) or alemtuzumab. No patients in our cohort had uncontrolled acute GvHD grades III-IV at time of vaccination. Two patients received rituximab within 6 months before the first dose of vaccination and failed to produce detectable anti-S IgG antibodies after vaccination. Regarding ATG use, we observed significantly lower anti-S IgG responses in patients having received ATG as part of their conditioning (27/41, 66\%; median $183 \mathrm{U} / \mathrm{ml}$ ) compared with patients who did not receive ATG (21/22, 95\%; median $2500 \mathrm{U} /$ 
a
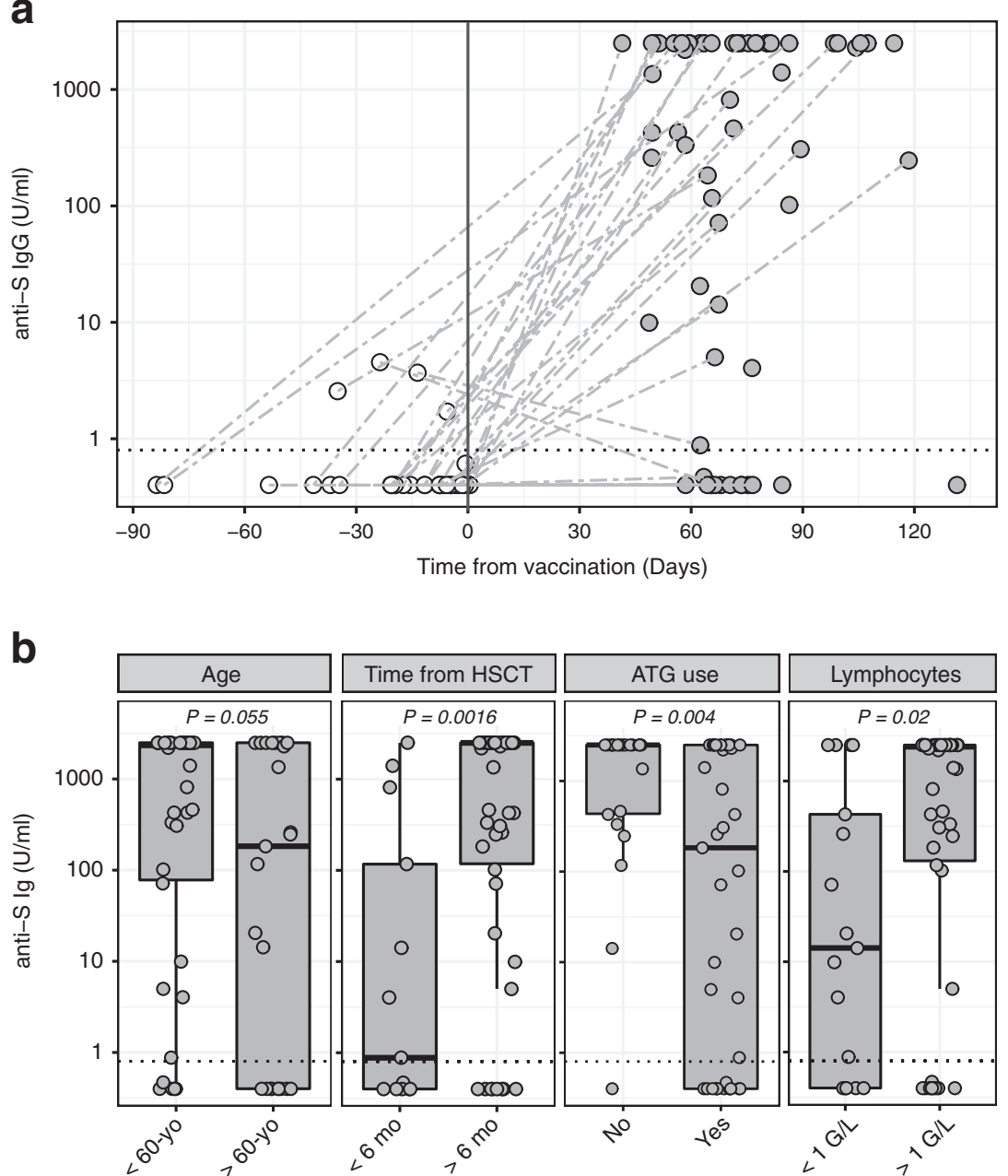

Fig. 1 Quantification of IgG against SARS-CoV-2 spike protein in response to vaccination in allogeneic HSCT recipients. a lgG against SARS-CoV-2 spike protein before and after mRNA-based SARS-CoV-2 vaccination (time 0). Dashed gray lines connect paired samples before and after mRNA-based SARS-CoV-2 vaccination in patients for which both samples were available. Dotted black line indicates the $0.8 \mathrm{U} / \mathrm{ml}$ positivity cutoff. $\mathbf{b}$ Levels of IgG against SARS-CoV-2 spike protein in allogeneic HSCT recipient stratified by age, time post-HSCT, ATG use during conditioning and lymphocyte count. Groups were compared using the non-parametric Mann-Whitney U test.

$\mathrm{ml} ; p=0.004$; Fig. 1b). Recently, Redjoul and colleagues reported an association between lymphocyte count and anti-S IgG responses in HSCT recipients [6]. Accordingly, we observed significantly higher anti-S lgG levels in patients with lymphocyte counts >1 G/L (37/46, 80\%; median $2396 \mathrm{U} / \mathrm{ml})$ compared with lymphopenic patients $(12 / 17,71 \%$; median $14 \mathrm{U} / \mathrm{ml} ; p=0.02$; Fig. 1b). Multiple logistic regression analysis confirmed the impact of patients' age, time since transplantation and ATG use while failed to confirm the impact of lymphocyte count on anti-S seroconversion (Supplementary Table 2).

Collectively, our data indicate that mRNA-based SARS-CoV-2 vaccination induced humoral responses in the majority of allogeneic HSCT recipients (76\%). Such a response rate, despite being lower than the one reported for the general population [3-5], is higher than the one reported in solid organ transplant recipients [7-10] and similar to the one described in patients with hematological cancers [11].

Focusing on clinical factors that are taken into account in clinical decision to vaccinate allogeneic HSCT patients according to the EBMT guidelines, we identified a short time (less than 6 months) after allogeneic HSCT and ATG use during conditioning as two factors negatively influencing anti-SARS-CoV-2 humoral responses to vaccination. These results suggest that delaying vaccination at later time-points whenever possible might improve immune responses by allowing for improved immunereconstitution and by potentially reducing the impact of lymphodepleting antigen employed during conditioning. Alternatively, providing a third dose of vaccine at later time points in patients not developing sufficient humoral responses to standard vaccination schedule might be an option as recently described in a small series of solid organ transplant recipients [12].

Our study has limitations including the limited number of subjects included and the absence of pre-vaccination samples in a significant proportion of patients. Moreover, induction of cellular responses was not assessed. More importantly, no correlate of protection for SARS-CoV-2 exist so far and only vaccine efficacy and effectiveness studies in this specific patients population will reveal if allogeneic HSCT recipients are protected against infection or severe disease by mRNA-based SARS-CoV-2 vaccines.

In conclusion, we observed a $76 \%$ rate of antibody responses to mRNA-based SARS-CoV-2 vaccination after allogeneic HSCT and we identified age, time since transplantation, and ATG use as three factors potentially affecting the response. After validation in larger patient cohorts, these results will help orient clinical management of SARS-CoV-2 prevention strategies in allogeneic HSCT recipients. 


\begin{abstract}
Anne-Claire Mamez (ID ${ }^{1,9 凶}$, Amandine Pradier ${ }^{1,2,9}$, Federica Giannotti ${ }^{1}$, Adrien Petitpas D $^{3}$, Marta Fabra Urdiola ${ }^{1}$, Diem-Lan Vu ${ }^{4}$, Stavroula Masouridi-Levrat (D) ${ }^{1}$, Sarah Morin ${ }^{1}$, Carole Dantin ${ }^{1}$, Dominique Clerc-Renaud ${ }^{1}$, Christiane S. Eberhardt ${ }^{5,6}$, Laurent Kaiser $4,7,8$, Federico Simonetta ${ }^{1,2,10}$ and Yves Chalandon (iD 1,2,10西 ${ }^{1}$ Division of Hematology, Department of Oncology, Geneva University Hospitals, University of Geneva, Geneva, Switzerland.

${ }^{2}$ Translational Research Centre in Onco-Haematology, Faculty of Medicine, University of Geneva, Geneva, Switzerland. ${ }^{3}$ Department of Political Science and International Relations, University of Geneva, Geneva, Switzerland. ${ }^{4}$ Division of Infectious Diseases, Geneva University Hospitals, 1205 Geneva, Switzerland. ${ }^{5}$ Center for Vaccinology, University Hospitals of Geneva and Division of General Pediatrics, Department of Woman, Child and Adolescent Medicine, Faculty of Medicine, University of Geneva, Geneva, Switzerland.

${ }^{6}$ Emory Vaccine Center, Emory University, Atlanta, GA, USA. ${ }^{7}$ Laboratory of Virology, Division of Infectious Diseases and Division of Laboratory Medicine, University Hospitals of Geneva \& Faculty of Medicine, University of Geneva, 1205 Geneva, Switzerland. ${ }^{8}$ Geneva Centre for Emerging Viral Diseases, Geneva University Hospitals, 1205 Geneva, Switzerland. ${ }^{9}$ These authors contributed equally: Anne-Claire Mamez, Amandine Pradier. ${ }^{10}$ These authors jointly supervised this work: Federico Simonetta, Yves Chalandon. ${ }^{凶}$ email: anne-claire. mamez@hcuge.ch; yves.chalandon@hcuge.ch
\end{abstract}

\section{REFERENCES}

1. Ljungman P, de la Camara R, Mikulska M, Tridello G, Aguado B, Zahrani MA, et al. COVID-19 and stem cell transplantation; results from an EBMT and GETH multicenter prospective survey. Leukemia. 2021. https://doi.org/10.1038/s41375-02101302-5.

2. Sharma A, Bhatt NS, Martin AS, Abid MB, Bloomquist J, Chemaly RF, et al. Clinical characteristics and outcomes of COVID-19 in haematopoietic stem-cell transplantation recipients: an observational cohort study. Lancet Haematol. 2021;8: e185-e193.

3. Walsh EE, Frenck RW, Falsey AR, Kitchin N, Absalon J, Gurtman A, et al. Safety and immunogenicity of two RNA-based Covid-19 vaccine candidates. N Engl J Med. 2020;383:2439-50.

4. Anderson EJ, Rouphael NG, Widge AT, Jackson LA, Roberts PC, Makhene M, et al. Safety and immunogenicity of SARS-CoV-2 mRNA-1273 vaccine in older adults. $\mathrm{N}$ Engl J Med. 2020;383:2427-38.

5. Baden LR, El Sahly HM, Essink B, Kotloff K, Frey S, Novak R, et al. Efficacy and safety of the mRNA-1273 SARS-CoV-2 vaccine. N. Engl J Med. 2021;384:403-16.

6. Redjoul R, Le Bouter A, Beckerich F, Fourati S, Maury S. Antibody response after second BNT162b2 dose in allogeneic HSCT recipients. Lancet. 2021;398:298-9.

7. Boyarsky BJ, Werbel WA, Avery RK, Tobian AAR, Massie AB, Segev DL, et al. Antibody response to 2-dose SARS-CoV-2 mRNA vaccine series in solid organ transplant recipients. JAMA. 2021;325:2204-6.
8. Marinaki S, Adamopoulos S, Degiannis D, Roussos S, Pavlopoulou ID, Hatzakis A, et al. Immunogenicity of SARS-CoV-2 BNT162b2 vaccine in solid organ transplant recipients. Am J Transplant. 2021. https://doi.org/10.1111/ajt.16607.

9. Grupper A, Rabinowich L, Schwartz D, Schwartz IF, Ben-Yehoyada M, Shashar M, et al. Reduced humoral response to mRNA SARS-CoV-2 BNT162b2 vaccine in kidney transplant recipients without prior exposure to the virus. Am J Transplant. 2021. https://doi.org/10.1111/ajt.16615.

10. Sattler A, Schrezenmeier E, Weber UA, Potekhin A, Bachmann F, StraubHohenbleicher $\mathrm{H}$, et al. Impaired humoral and cellular immunity after SARS-CoV2 BNT162b2 (Tozinameran) prime-boost vaccination in kidney transplant recipients. J Clin Investig. 2021;131:e150175.

11. Monin L, Laing AG, Muñoz-Ruiz M, McKenzie DR, Del Molino Del Barrio I, Alaguthurai $\mathrm{T}$, et al. Safety and immunogenicity of one versus two doses of the COVID-19 vaccine BNT162b2 for patients with cancer: interim analysis of a prospective observational study. Lancet Oncol. 2021;22:765-78.

12. Werbel WA, Boyarsky BJ, Ou MT, Massie AB, Tobian AAR, Garonzik-Wang JM, et al. Safety and immunogenicity of a third dose of SARS-CoV-2 vaccine in solid organ transplant recipients: a case series. Ann Intern Med. 2021. https://doi.org/ 10.7326/L21-0282.

\section{AUTHOR CONTRIBUTIONS}

ACM, A Pradier, FS and YC conceived and designed the study; ACM, A Pradier, FG, MFU, CD and DCR collected the data; ACM, A Pradier, A Petitpas, and FS analyzed and interpreted the data; FG, DLV, SML, SM, CSE, LK and $Y C$ contributed to the interpretation of the data; ACM, A Pradier, FS and YC wrote the manuscript; FS and YC supervised the research; all authors read, edited, and approved the submitted version of the manuscript.

\section{COMPETING INTERESTS}

The authors declare no competing interests.

\section{ADDITIONAL INFORMATION}

Supplementary information The online version contains supplementary material available at https://doi.org/10.1038/s41409-021-01466-9.

Correspondence and requests for materials should be addressed to Anne-Claire Mamez or Yves Chalandon.

Reprints and permission information is available at http://www.nature.com/ reprints

Publisher's note Springer Nature remains neutral with regard to jurisdictional claims in published maps and institutional affiliations. 